V.

Aus dem pharmakologischen Institut zu Marburg.

\title{
Ueber den Einfluss des Camphers auf die Grösse der Zuckerausscheidung im Phlorhizindiabetes.
}

Ein Beitrag zur Lehrevon der Herkunft der Glueuronsäure im Thierkörper.

Vort

Dr. Otto Loowi,

Privatdocent und Assistent des Institutes.

\section{Einleitung.}

Die an die Entdeckung des Phlorbizindiabetes gekntipfte Hoffnung, in Art und Grösse der Zuckerbildung im Thierkörper Einblick zu erlangen, hat sich bisher nur zum geringsten Theil erfullt. Der Grund hierfür mag darin liegen, dass wir erst in jutngster Zeit durch Untersuchungen von $\mathrm{Creme} \mathrm{r}^{1}$ ) und von $\mathrm{Lusk}^{2}$ ) die Bedingungen kennen lernten, unter denen die Znckerausscheidung. phlorhizinbehandelter Thiere gleichmässig abläuft. Die von den genannten Forschern getibte Methodik schien mir fur die Prüfung der Frage nach dem Zusammenbang von Zueker- und Glucuronsäurebildung anwendbar.

Unsre Kenntnisse über die Bildungsart der Gluouronsäure im thierisehen Organismus sind sebr dïftig. Sch miedeberg u. Meyer ${ }^{3}$ ) hielten die Säure für ein Oxydationsproduct der Glucose, der sie chemisch nahe steht. Indess liegt ein Beweis für diese Annahme nicht vor. Aber wäre selbst nachgewiesen, dass nach Einfuhr eines Glueuronsäurepaarlings oder dessen Vorstufe die Glucuronsäure aus Glucose entsteht, so wäre daraus die Verallgemeinerung, dass

1) Cremer u. Ritter, Zeitschr. f. Biol. Bd. 29. S. 256. 1893.

2) Americ. journ. of physiol. V. T. S. 396. 1898.

3) Zeitschr. f. physiol. Chem. Bd. 3. S. 422.1879. 
der Abbau der Glucose auch in der Norm auf diese Weise vor sich geht, keineswegs abzuleiten. Eine solche Annahme wäre sogar unwahrscheinlich. Es ist nämlich nicht einzusehen, wie bei der Oxydation der Glucose die Aldehydgruppe unverändert bleiben sollte, während die endständige Alkoholgruppe in Carboxyl ubergeht. Dies wäre nach Emil Fischer 1) nur dann möglich, wenn von vornherein die Aldehydgruppe des Zuckers dureh Paarung festgelegt und so vor weiterer Oxydation geschutzt wird. Deshalb nimmt Emil Fischer auch an, dass bei der Bildung gepaarter Glucuronsäuren die Paarung nicht mit der fertig gebildeten Säure sondern bereits mit der Glucose statthat, wobei deren Aldehydgruppe festgelegt wird. Als weitere Folge ergiebt sich dann, dass nur beim Vorhandensein eines Paarlings die Oxydation der Glucose über Glucuronsäure geht.

Jüngst hat nun $\mathrm{Paul} \mathrm{Mayer}{ }^{2}$ ) angegeben bei den verschiedensten Krankheiten gepaarte Glucuronsäuren z. T. in erheblichen, Mengen gefunden zu haben. Der Autor geht von der Hypothese, dass die Oxydation der Glucose über Glucuronsäure geht, als von einer feststehenden Thatsache aus und deutet seine Befunde so, dass der Glucuronsäure ausscheidende Organismus noch die Fähigkeit besitze, Glucose zu Glucuronsäure zu oxydiren, aber nicht mehr diese weiter zu verbrennen. Ersteres sei für den Organismus leichter als letzteres. Ueber das plötzliche Auftreten des Paarlings äussert sich der Autor nicht. Da aber nach allen vorliegenden Erfahrungen allein diese Bedingung für die Bildung von Glucuronsäure primär verantwortlich zu machen ist, mlissen wir bis auf weiteres auch in den von P. Mayer mitgetheilten Fällen in dem, sei es durch Darmfäulniss oder sonstwie bewirkten Auftreten des Paarlings, nicht aber in herabgesetzter Oxydationskraft des Organismus den Grund für das Auftreten von Glucuronsäure suchen ${ }^{3}$ ).

An eine ganz andere Quelle der Glucuronsäure als an die Glucose dachte Schmiedeberg ${ }^{4}$ ) in einer späteren Arbeit. Er erhielt nämlich bei der Spaltung der Chondroitinschwefelsäure unter anderem eine Säure, die er als Glucuronsäure ansprechen zu dürfen

1) Ber. d. d. chem. Gesch. Bd. 24. S. 524. 1891.

2) Dtsch. med. Wchschr. Nr. 16, 17. 1901.

3) Anm. b. d. Corr. Blumenthal, Verhandlungen der physiol. Gesellsch. zu Berlin. Jahrg. 1900-1901. Nr. 14-17. 1. August 1901.

4) Arch. f. exp. Path. u. Pharm. Bd. 28. S. 355. 1891. 
glaubte, und erörterte die Möglichkeit, dass diese das Material für den Paarling des Camphers und andrer Stoffe abgebe. War eș nicht leicht, sich vorzustellen, dass der gefässarme Knorpel in so intensiver Weise sich am Gesammtstoffwechsel betheilige, so war der.

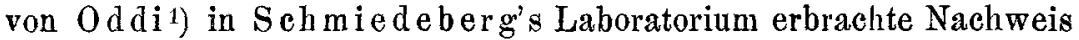
von Chondroitinscliwefelsäure in der Leber einer solehen Auffassung um so güunstiger.

Möglicherweise entsteht aber die Glucuronsäure noch auf ganz andere, bisher nicht berẗcksichtigte Weise, nämlich aus einem Theil des Eiweisses, der mit der Zuckerbildung gar nichts zu thun hat.

Bei dieser Sachlage bedarf die Anstellung von Versuchen zur Klärung dieser Frage keiner Rechtfertigung.

Nach folgendem Gedankengang schien mir eine Entscheidung, wenigstens in Bezug auf die erstangefuhrte Hypothese sich anbahnen zu lassen. Es gelingt, wie erwähnt, nach einer in der Hauptsache von Lusk ausgearbeiteten Methode, bei Hunden durch Phlorhizin, eine gleichmässige, hohe Zuckerausscheidung zu bewirken, die insofern eine totale $\mathrm{zu}$ nennen ist, als weder klinisoh noch experimentell auf andrem Wege eine gleich hohe oder höhere "Zuckeransscheidung beobachtet wird. Unter dieser und zwar nur unter dieser Voraussetzung, dass nämlich bei der maximalen Phlorhizinvergiftung keine Zuckervorstufe oder Zucker selbst im Körper verbraucht und deshalb nicht ausgeschieden wird, gilt die folgende Ueberlegung und Versuchsanordnung:

Führt man einem mit Phlorhizin entsprechend behandelten Thier einen Körper za, der sich mit Glucuronsäure paart, so muss die Zuckerausscheidung um den zur Bildung der Glucuronsäure nöthigen Betrag fallen, sofern die Muttersubstanzen für Zucker und Glucuronsäure dieselben sind, bez. die Säure aus dem Zueker hervorgeht. Andernfalls wird die Grösse der Znckerausseheidung durch Einführung eines Glucuronsäurebildners nicht beeinflusst werden.

Zunächst bedarf die Anwendung von Phlorhizin zur Entscheidung von Stoffwechselfragen der Rechtfertigung, da hiergegen wegen des angeblich pathologischen Verhaltens der phlorhizinbehandelten Thiere verschiedentlieh Bedenken erhoben wurden.

Halsey ${ }^{2}$ ) beobachtete gelegentlioh bei seinen hier im Laboratorium angestellten Versuchen Krämpfe. Gleichzeitig traten Abscesse

1) Arch. f. exp. Păils. u. Pharm. Bủ. 33̆. S. 376. 1894.

2) Sitzungsber. der Ges. z. Bef. d. ges. Nat. Nr. 5. 1899. 
auf, ein Vorkommniss, das auch Hartogh u. Schumm ${ }^{1}$ ) angeben. v. Kossa ${ }^{2}$ ) will regelmässig Nephritis mit Albuminurie beobachtet haben.

Selbstverständlich können die Ergebnisse von Untersuchungen an nephritischen, eiternden, in Krämpfen liegenden Thieren nur einen beschränkten Werth für die Beurtheilung physiologischer Vorgänge b eanspruchen.

Ausser in Versuch 1, wo es zu Abscessbildung kam, habe ich nicht in einem einzigen Falle irgend eine der oben erwähnten Erscheinungen beobachtet und doch wurden die Thiere z. T. solange unter ständiger Phlorhizinwirkung gehalten wie nie in Versuchen zuvor.

\section{Versuchsanordnung.}

Zur Injection diente eine $35 \mathrm{ccm}$ fassende Glasspritze, die vor und nach Benutzung mit officineller Phenollösung öfters ansgespult wurde. Mit der Injection wurde erst begonnen, nachdem ich mich durch leichte Verschieblichkeit der Spitze darüber vergewissert hatte, dass die Spitze nicht in der Haut sondern im Unterhautzellgewebe sich befand. Dann und nur dann ging die Injection leicht und ohne Widerstand vor sich. Durch gleichzeitiges leichtes Massiren der Injectionsstelle wurde die Anhäufung der Injectionsflüssigkeit hintangehalten, die nach früher im Laboratorium gewonnenen Erfahrungen leicht zur Bildung von Abscessen führt. Die Injection ist unter diesen Umständen absolut schmerzlos. Schreien die Thiere, so ist dies auf den Einstich oder auf fehlerhaftes Injectionsverfahren zu beziehen.

Das Phlorhizin (Merck) wurde erst vor der Injection in 1 proc. Lösung von Natriumcarbonat und zwar $1 \mathrm{~g}$ in $20 \mathrm{ecm}$ gelöst und nach Erwärmung auf $37^{\circ}$ injicirt.

Die Injection wurde in 8 stündigen Intervallen ausgeführt. Für jedes Thier wurde die Dose bestimmt, bei deren Steigerung eine Erhöhung der' Zuckerausfuhr nicht mehr stattfand.

Eine gewisse Schwierigkeit bietet die Wahl geeigneter Körper, die sich mit Glucuronsäure paaren, da die meisten in grösseren Dosen, und um die Einfuhr soleher handelt es sich, wenn deutliche Ausschläge erzielt werden sollen, für den Organismus nicht indifferent sind und die Zuckerausseheidung aus Gründen beeinflussen können, die mit der Glucuronsäurebildung als solcher gar nichts zu thun zu haben brauchen.

Ich wählte den Campher, da die duroh ihn gesetzte Schädigung gering ist und die Thiere sich leicht an ihn gewöhnen, sodass er

1) Ds. Arch. Bd. 45. S. 11.1900.

2) Ztschr. f. Biol. Bd. 4\%. S. 324. 1900. 
in grösseren Dosen gereicht werden kann. Er wurde, feinpulverisiert, in Duten aus japanischem Papier den Tieren eingeschoben. Der Koth roch fast nie nach dem Mittel. Die Resorption ist demnach eine sehr vollständige. Es ist unwahrscheinlich, dass sie lediglich nach Maassgabe seiner Flüchtigkeit stattfindet; ich möchte sie eher auf dieselbe Weise erklären, wie ich das jüngst für die Resorption des Anthracens gethan habe 1), nämlich durch Lösung in den Darmepithelien.

$\mathrm{Zu}$ den Versuchen wurden Hündinnen benutzt, die nach Falck für das Katheterisiren vorbereitet waren. Die Thiere wurden in Käfigen gehalten, die eine peinliche Sammlung des Harns ermöglichten. Es wurde mit Wasser nachgespült.

Als Nahrung diente, wo nichts Besonderes bemerkt ist, gewiegtes Rindfleisch, dessen N-Gehalt nach Ausweis zahlreicher Analysen zwischen $3,2+3,4$ Proc. schwankte.

Der Harn wurde alle 24 Stunden entnommen und auf ein bestimmtes Volum mit destillirtem Wasser aufgefüllt. Die in den Versuchen niedergelegten Zahlen für den Procentgehalt beziehen sich auf dieses.

Der Stickstoff wurde nach $\mathrm{Kj}$ eld a hl, der Zucker in allen Versuchen durch Drehung vor and nach Vergährung bestimmt. In einem Versuch wurde daneben nach Fehling (genau entsprechend den Angaben von Huppert ${ }^{2}$ ), in einem anderen nach Lehmann ${ }^{3}$ ) titrirt, in mehreren schliesslich wurde die Wägungsmethode nach Allihn befolgt. Die Phosphorsäure wurde durch Titration mit Uranlösung ermittelt.

Sämmtliche Bestimmungen wurden mindestens doppelt ausgeführt.

Die Menge der ausgeschiedenen Camphoglucuronsäuren berechnete ich aus der Linksdrebung des Harns nach der Vergährung. Der Berechnung wurde das Drehungsvermögen der d Camphoglucuronsäure zu Grunde gelegt, was insofern willkurlich ist, als nach Schmiedeberg u. Meyer noch 2 andere Säuren nach Campherfütterung ausgeschieden werden, deren Drehungsvermögen nicht feststeht. Da es aber nicht auf eine exacte Bestimmung ankam, schien mir das Verfahren ausreichend.

Die Berechnung ist folgende: Da sich das Drehungsvermögen der GIucose $(52,5)$ zu dem der d Camphoglucuronsäure (- 32,85) wie $1,6: 1$ verhält, entspricht ein Theilstrich der Saccharimeterscala $1,6 \%$ d Camphoglucuronsäure.

Ferner bedarf es zur Bildung von 1 g Camphoglucuronsäure $0,54 \mathrm{~g}$ Glucuronsäure bez. $0,5 \mathrm{~g}$ Glucose.

1) Sitzgsber. d. Ges. z. Bef. d. ges. Nat. Nr. 7. 1901.

2) Anaiyse des Harns. 1898.

3) Arch. f. Hyg. Bd. 30. S. 267. 1898. 


\section{Versuche.}

Versuch 1 . Phlorhizin.

Weiblicher Schäferhund $17,0 \mathrm{~kg}$ schwer. $3 \mathrm{mal}$ täglich $1,5 \mathrm{~g}$

Futter: $500 \mathrm{~g}$ Fleisch, ad libitum Wasser.

\begin{tabular}{|c|c|c|c|c|c|c|c|c|c|c|c|}
\hline $\begin{array}{c}\text { Datum } \\
1899\end{array}$ & $\begin{array}{c}\text { Harn- } \\
\text { menge } \\
\text { cem }\end{array}$ & $\begin{array}{l}N \\
g\end{array}$ & $\begin{array}{l}\% \text { \% } \\
\text { vor } \\
\text { Verg } \\
\end{array}$ & $\begin{array}{r}\text { Zuc } \\
\text { Pol. }{ }^{1} \\
\text { [nach } \\
\text { ahrg. }\end{array}$ & $\begin{array}{l}\text { ker } \\
\text { abs } \\
\text { Pol. }\end{array}$ & $\begin{array}{l}\text { olut } \\
\left(\operatorname{Red}^{2}\right)\end{array}$ & 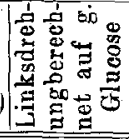 & $\begin{array}{l}\ddot{\sigma} \\
\ddot{a}\end{array}$ & $\mathrm{P}_{2} \mathrm{O}_{5}$ & 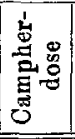 & 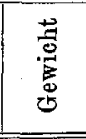 \\
\hline 8. XI. & 710 & 29,60 & 4,8 & 0,2 & 98,4 & 99,0 & & 3,4 & 5,03 & & $17,0^{\circ}$ \\
\hline 9. & 830 & 24,32 & 4,5 & 0,2 & 93,8 & 98,9 & & 3 & & & \\
\hline 10. & 850 & 25,20 & 4,7 & 0,2 & 97,0 & 102,0 & & 3 & 3,70 & & 16,5 \\
\hline 11. & 790 & 24,01 & 4,3 & 0,2 & 89,2 & 94,4 & & 3 , & 3,61 & & 16,65 \\
\hline 12. & 800 & 18,08 & 3,2 & 0,8 & 74,4 & 77,0 & 11,2 & 4 & & $20 \mathrm{~g}$ & 15 \\
\hline 13. & 890 & 18,80 & 1,1 & 1,3 & 47,8 & 54,0 & 17,6 & 2, & 2,36 & & 15,65 \\
\hline 14. & 680 & 26,8 & 2,2 & 0,8 & 58,2 & 62,6 & 9,6 & $\mathbf{2}$, & 2,50 & ló $\mathrm{g}$ & 15,4 \\
\hline & 750 & & 3,3 & 0,2 & & 66,6 & & 3, & 2,1 & & \\
\hline 16 & 910 & 21,12 & 3,1 & 0,2 & 65,6 & 73,4 & & 3,1 & 2,20 & & 15,1 \\
\hline
\end{tabular}

\section{Bespreohung des Versuehs.}

Es wurde solange mit der Campherdarreichung gewartet, bis sich ein constantes Verhältniss zwischen Glucose- und Stickstoffausscheidung (D:N) eingestellt hatte. Eine Complication trat dadurch ein, dass die bis dahin sehr gleichmässige $\mathrm{N}$-Ausscheidung durch die Campherdarreichung nicht unbeträchtlich vermindert wurde. Es handelt sich dabei nicht um eine Behinderung der Nahrungsresorption durch den Campher, da in beiläufig dieserhalb angestellten Untersuchungen selbst nach hohen Camphergaben eine NVermehrung im Koth nicht gefunden wurde. Auch für die Annahme einer Ausscheidungsverzögerung liegen keine Anhaltspunkte vor, erstlich ist die Harnmenge nicht geringer wie sonst, ferner findet keine Nachausscheidung von $\mathrm{N}$ statt.

Mit der N-Ausscheidung sinkt auch die des Zuckers, jedoch am 12. XI. trotz Auftretens von Glucuronsäure in geringerem Maasse als die des Stickstoffs, sodass $D: N$ sogar etwas ansteigt (von 3,8

1) Der auf $2000 \mathrm{ccm}$ aufgefüllten Tagesmenge.

2) Allihn.

3) lv sämmtlichen Versuchen wird der Berechnung des Factors die durch Polarisation erhaltene Zuckermenge zu Grunde gelegt. 
auf 4,1). An den nächsten Tagen freilich sinkt die Zuckerausscheidung, sodass $\mathrm{D}: \mathrm{N}$ fällt. Bemerkenswerther Weise erreicht $\mathrm{D}: \mathrm{N}$ aber auch nach dem Abklingen der Glucuronsäureausscheidung: seine frtihere Höhe nicht mehr, was der Fall sein musste, wenn das Absinken der Zuckerausscheidung nur durch die Glucuronsäurebildung veranlasst gewesen wäre; wir sind demnach gezwungen, in diesem Versuche eine von der Glucuronsäurebildung unabhängige Beeinflussung der Zuckerausscheidung durch Campher anzunehmen. $\mathrm{Ob}$ die Glucuronsäurebildung an der Zuckerverminderung betheiligt ist, lässt sich zwar in diesem Versuch nicht sicher ausschliessen, ist aber bis zu einem gewissen Grad unwahrscheinlich, da am ersten Tage trotz der gleichzeitigen, beträchtlichen Glucuronsäureausfuhr im Verhältniss zum Stickstoff sogar mehr Zucker im Harn auftrat, als vorher ohne Campher.

Versueh 2 .

Weiblicher Schäferhund $21,0 \mathrm{~kg}$ schwer. Versuchsanordnung wie oben.

\begin{tabular}{|c|c|c|c|c|c|c|c|c|c|c|c|}
\hline $\begin{array}{c}\text { Datum } \\
1899\end{array}$ & $\mathrm{~N}$ & $\begin{array}{l}\% \% \\
\text { v. Ver- } \\
\text { gährg. }\end{array}$ & $\begin{array}{l}\text { Zucker } \\
\% / 0 \\
\text { n. Ver } \\
\text { gährg. }\end{array}$ & $\begin{array}{c}\text { abso- } \\
\text { lut }\end{array}$ & 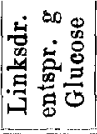 & $\begin{array}{l}z \\
\ddot{a}\end{array}$ & 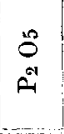 & 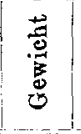 & Nahrung & $\stackrel{\dot{\vec{g}}}{\vec{E}}$ & Bemerkungen \\
\hline 6.XII. & 23,8 & 4,7 & 0,2 & 98,0 & & 4,1 & 1,88 & 21,09 & $500 \mathrm{~g} \mathrm{Fl}$ & $38, \tilde{5}$ & \\
\hline & $25,97 \mid$ & 5,2 & 0,3 & 110,0 & & 4,2 & 2,84 & 20,80 & $=$ & & \\
\hline 8. & 33,60 & 5,1 & 0,2 & 106,0 & & 3,1 & 4,16 & 21,05 & $750 \mathrm{~g} \mathrm{Fl}$ & & \\
\hline 9 & 29,68 & 4,3 & 0,2 & 90,0 & & 3,0 & 3,32 & 20,92 & $=$ & & \\
\hline 10. & 15,76 & 2,7 & 0,2 & 58,0 & 21,0 & 3,8 & 2,66 & $21,0 \mid$ & $=$ & & $20 \mathrm{~g}$ Campher \\
\hline 11. & 31,08 & 2,7 & 1,5 & 84,0 & 8,0 & 2,7 & 3,62 & 20,92 & $=$ & & \\
\hline 12. & 32,65 & 3,0 & 0,7 & 74,0 & & 2,2 & 3,04 & 20,74 & $=$ & & \\
\hline 13. & - & - & - & - & & - & - & - & $=$ & & Harn unvollstg. \\
\hline 14. & 19,38 & 2,5 & 0,2 & 54,0 & & 2,8 & 2,30 & 20,77 & $=$ & & \\
\hline 15. & 19,20 & 2,9 & 0,1 & 60,0 & & 3,1 & 2,54 & 20,85 & $=$ & & \\
\hline 16. & 22,12 & 2,9 & 0,2 & 62,0 & & 2,8 & 2,74 & 20,94 & $=$ & & \\
\hline 17. & 22,57 & 2,9 & 0,3 & $6 \mathrm{l}, 0$ & & 2,9 & 2,62 & 21,05 & $=$ & 38,3 & \\
\hline 18. & 21,0 & 2,6 & 0,2 & 55,0 & & 2,7 & 2,44 & 20,60 & $=$ & 39,5 & \\
\hline 19. & 20,89 & 2,6 & 0,7 & 66,0 & 8,0 & 3,1 & 2,06 & $20,05 \mid$ & $=$ & 38,5 & 25g Campher \\
\hline 20. & 25,37 & 1,3 & 1,9 & 64,0 & 28,0 & 2,6 & 2,30 & $|20,55|$ & $=$ & 38,8 & \\
\hline 21. & 34,80 & 3,1 & 0,2 & 66,0 & & 1,9 & 2,75 & 20,55 & $=$ & 38,4 & \\
\hline 23. & 28,77 & 3,0 & 0,3 & 66,0 & & 2,3 & 2,82 & 20,50 & $=$ & 39,0 & \\
\hline 22. & 25,46 & 2,7 & 0,2 & 58,0 & & 2,3 & - & $|20,52|$ & $=$ & 38,8 & \\
\hline
\end{tabular}

Besprechung des Versuchs.

Auch in diesem Versuch sank nach der ersten Campherdarreichung (10. XII.) der Stickstoff beträchtlich ab. Die Zuckerausscheidung verhält sich ähnlich wie in Versuch I d. h. sie sinkt 
weniger als der Stickstoffausscheidung entspricht, sodass das Verhältniss $D: N$ am ersten Tage ansteigt, um dann etwas abzusinken, deutlich erst am 3. Tage nach der Campherdarreichung. Am 2. Tage, an dem die grösste Glucuronsäuremenge ausgeschieden wurde, erreichte sowobl die absolute Zuckermenge wie die in Relation zum $\mathrm{N}$ ausgeschiedene annähernd denselben Stand wie früher. Und doch hätte, wie die folgende Berechnung lehrt, die Zuckerausscheidung an diesem Tage beträchtlich geringer sein müssen, falls Vicariiren mit der Glucuronsäure stattgefunden hätte.

\section{Berechnung.}

11. XII. Linksdrehung entspr. $1,5-0,2=1,3$ Proc. Glucose

$=2,1=$ Camphoglucuronsäure bei einer Harnm. v. $2000=42 \mathrm{~g}$

Diese beanspruchen für ihre Bildung $21 \mathrm{~g}$ Glucose, die weniger ausgeschieden hätten werden mtissen, statt dessen wurden nur $6 \mathrm{~g}$ weniger ausgeschieden als vorher.

Also auch dieser Versuch spricht gegen eine Beziehung zwischen Zucker- und Glueuronsäurebildung. Ganz eindeutig beweist dies aber der Erfolg der näehsten Camphergabe, die erst verabfolgt wurde, als sich D:N und die absolnten Ausscheidungen wieder mehrere Tage constant eingestellt hatten. Trotz einer Glucuronsäureausscheidung, die einer Zuckermenge von ca. $8 \mathrm{~g}$ entspricht, und trotz gleichbleibender Stickstoffzahl, steigt hier am ersten Tag. (19. XII.) der Zucker um $10 \mathrm{~g}$ an, sodass auch das Verhältniss D : N ansteigt. Am nächsten Tag (20. XII.), an dem die ausgeschiedene Glucuronsäure einer Zuckermenge von ca. $28 \mathrm{~g}$ entspricht, hielt sich die Zuckerausscheidung auf derselben Höhe, D:N erreicht den früheren Werth wieder.

Hieraus scheint mit Sicherheit hervorzugehen, dass die Glucuronsäurebildung nichts mit der Zuckerbildung zu thun hat.

An den folgenden Tagen, an denen gar keine Glucuronsäure mehr erschien, sank $\mathrm{D}: \mathrm{N}$ wieder ab. Dabei blieb jedoch die absolute Menge des ausgeschiedenen Zuckers auf der bisherigen Höhe; das Sinken von $D: N$ ist auf das Ansteigen des Stickstoffs zu beziehen. 
Versuch 3.

Derselbe Hund. $500 \mathrm{~g}$ Fleisch. $\mathrm{H}_{2} \mathrm{O}$ ad libitum.

\begin{tabular}{|c|c|c|c|c|c|c|c|c|c|c|c|c|c|}
\hline \multirow[b]{2}{*}{ No. } & \multirow[b]{2}{*}{$\begin{array}{c}\text { Datum } \\
1900\end{array}$} & \multirow{2}{*}{ 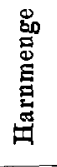 } & \multirow[b]{2}{*}{$\mathrm{N}$} & \multicolumn{2}{|c|}{ Zucker } & \multirow[b]{2}{*}{ 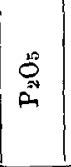 } & \multirow[b]{2}{*}{$\begin{array}{l}\stackrel{\Delta}{\circ} \\
\stackrel{\sim}{z}\end{array}$} & \multirow[b]{2}{*}{$\begin{array}{l}z \\
\ddot{\theta}\end{array}$} & \multirow{2}{*}{ 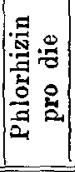 } & \multirow[b]{2}{*}{$\begin{array}{l}\text { Bemer- } \\
\text { kungen }\end{array}$} & \multirow{2}{*}{\multicolumn{2}{|c|}{$\begin{array}{c}\text { Linksdrehung } \\
\text { berechnet auf } \\
\text { Glucose } \\
\text { Proc.| absolut }\end{array}$}} & \multirow[b]{2}{*}{ 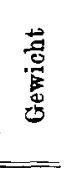 } \\
\hline & & & & 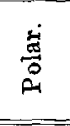 & $\widetilde{\vec{\sigma}}$ & & & & & & & & \\
\hline 1 & 9. $\mathrm{V}$ & 800 & 21,88 & 80,8 & & 2,18 & 9,95 & 3,69 & 4,5 & & & & 25,2 \\
\hline 2 & 10. & & 24,23 & & & 2,75 & 8,64 & 3,56 & $=$ & & & & 25,3 \\
\hline 3 & 11. & 840 & 24,18 & $80, T$ & & 2,61 & 9,3 & 3,33 & $=$ & & & & 25,05 \\
\hline 4 & 12. & 780 & 18,87 & 66 & & 2,41 & 7,87 & 3,5 & $=$ & & & & - \\
\hline 5 & 13. & & 18,43 & & & 2,12 & 8,76 & 3,8 & $=$ & & & & 24,3 \\
\hline 6 & & 70 & 19,10 & 68 & & 2,35 & 7,96 & 3,6 & $=$ & & & & 24,0 \\
\hline 7 & 15. & 850 & 19,66 & 68 & & 2,38 & 8,2 & 3,48 & $=$ & & & & 24,05 \\
\hline 8 & 16. & & 19,6 & 70 & & & $\mathbf{9 , 3}$ & 3,6 & $=$ & & & & 23,7 \\
\hline 9 & 17. & 730 & 20,16 & 68 & & 2,38 & 8,4 & 3,4 & $=$ & & & & 23,65 \\
\hline 10 & 18. & 730 & 19,77 & 72 & & 2,23 & 9,0 & 3,66 & $=$ & & & & 23,53 \\
\hline 11 & 19. & & 19 & & & 9 & 8,4 & 3,55 & $=$ & & & & 23,35 \\
\hline 12 & 20. & & 19,66 & 69 & & 2,03 & 9,85 & 3,52 & 7,5 & & & & 23,20 \\
\hline 13 & 21. & 710 & 17,02 & 60,8 & 60,1 & 2,38 & 7,01 & 3,58 & 4,5 & & & & 23,03 \\
\hline 14 & 22. & 790 & 19,4 & 64 & 61,0 & 1,96 & $9,7 \mathbf{5}$ & 3,32 & $=$ & & & & 23,0 \\
\hline 15 & 23. & 70 & 17,9 & 56,2 & 58,6 & 1,76 & 10,0 & 3,14 & $=$ & & & & 22,7 \\
\hline 16 & 24. & 650 & 16,70 & 55,4 & 56,7 & 1,86 & 8,8 & 3,31 & $=$ & & & & 22,57 \\
\hline 17 & 25. & 780 & 18,5 & 60,8 & 57,8 & 1,87 & 9,8 & 3,27 & $=$ & & & & 22,4 \\
\hline 18 & 26. & 610 & 16,18 & 53,8 & & 2,00 & 8,9 & 3,32 & $=$ & & & & 22,42 \\
\hline 19 & 27. & 710 & 15,84 & 50,7 & & 1,65 & 9,35 & $3,21\}$ & $=$ & & 0,2 & & 22,12 \\
\hline 20 & 28. & 810 & 16,02 & 52,3 & & 2,38 & 6,7 & 3,27 & $=$ & & $\begin{array}{l}1,25 \\
0,33\end{array}$ & $\begin{array}{r}17,4 \\
2,6\end{array}$ & 22,0 \\
\hline 21 & 29. & 750 & 18,59 & 45,5 & & 2,10 & 8,86 & 2,44 & $=$ & & 0,2 & & 21,8 \\
\hline 22 & 30. & & 18,1 & 49 & & 2,04 & 9,0 & 2,71 & $=$ & & & & 21,65 \\
\hline 23 & 31. & 0 & 15, & 50 & & 4,76 & 8,72 & 3,23 & $=$ & & & & 21,5 \\
\hline 24 & 1. VI & 790 & 15 & 45 & & 1,88 & 8,22 & 2,9 & $=$ & & & & 21,5 \\
\hline 25 & 2. & & 15 & 56 & & 1,61 & 9,94 & 3,53 & $=$ & & & & 21,6 \\
\hline 26 & 3. & 73 & 21, & 71 & & 2,34 & 9,35 & 3,34 & $=$ & & & & 21,7 \\
\hline 27 & 4. & 910 & 21,81 & 66 , & & 2,08 & 10,4 & 3,07 & $=$ & & & & 22,07 \\
\hline 28 & 5 . & & 21,95 & 70,2 & & 2,21 & 10,0 & 3,19 & $=$ & & & & 217 \\
\hline 29 & 6. & 770 & 一 & - & & - & - & - & - & & & & 22,12 \\
\hline 30 & 7. & 850 & & & & 100 & 8.15 & 3,11 & & & & & 21,80 \\
\hline 31 & 8. & 890 & 15 & 47. & & 1,99 & 7.6 & 3,15 & & & & & 22,0 \\
\hline 32 & 9. & 690 & 17,64 & 56, & & 1,77 & 9,8 & 3,23 & & & & & 21,27 \\
\hline 33 & 10. & 710 & 19,50 & 66,3 & & 2,67 & 7,2 & 3,4 & & & & & 15 \\
\hline 34 & 11. & 720 & $\mid 16,74$ & $\mathbf{3 3 , 5}$ & & 2,08 & 7,96 & $2,-$ & & $20 \mathrm{~g}$ & 1,25 & 17,4 & 20,85 \\
\hline & 12. & 850 & 16,02 & & & & 8,0 & 2,54 & & & 0,2 & & 20,6 \\
\hline 36 & 13. & 910 & 14,6 & 40 & & $1, i 2$ & 8,6 & 2,8 & & & & & 20,35 \\
\hline 37 & 14. & 720 & 12,6 & 42,9 & & 1,67 & 7,47 & 3,38 & & & & & 20,15 \\
\hline 38 & 15. & 850 & 19,19 & 49 & & - & & 2,56 & & & & & 20,25 \\
\hline 39 & 16. & 770 & 18,14 & 50,7 & & -1 & & 2,8 & & & & & 19,90 \\
\hline
\end{tabular}

1) Nach I ehmann; die Bestimmungen verdanke ich der Freundlichkeit ron Dr. J. T. Halsey. 


\section{Besprechung des Versuchs.}

Da versehiedentlich im Lauf der bisherigen Versuche das Verhältniss $\mathrm{D}: \mathrm{N}$ nach Campherdarreichung abgesunken war, der Campher aber erst gereicht wurde, nachdem schon längere Zeit das Thier mit Phlorhizin vorbehandelt war, war zu prufen, ob ev. eine Gewöhnung an Phlorhizin eintrete, oder ob der Campher das Sinken der Zuckerausseheidung veranlasse.

Deshalb wurde in diesem Versuch eine lange Vorreihe angestellt. Sie ergab, dass durch fortgesetzte Phlorhizinbehandlung eine Aenderung von $\mathrm{D}: \mathrm{N}$ nicht zu erzielen ist. ${ }^{1)}$ Stickstoff- und Zuckerausseheidung nehmen zwar mit der Zeit etwas ab, aber in demselben Verhăltniss.

Das Ergebniss der ersten Campherfutterung schliesst sich dem in dem vorigen Versuch gewonnenen gleichsinnig an. Was allerdings den Effect der zweiten Campherftitterung betrifft, so lässt diese eine von der bisherigen abweichende Deutung zu. Es ist aber $\mathrm{zu}$ bedenken, dass dureh den lange dauernden Versuch bereits derartige Ungleichmässigkeit in den Ausscheidungen sich zeigt, dass bindende Sohlüsse nicht mehr zu ziehen sind.

\section{Versuch 4.}

Derselbe Hund wie in Versuch 1 und 3.

Seit 18. V. 1901. Abends Hunger.

19. V. 1901. $3 \mathrm{~h} \mathrm{1.} \mathrm{Injection} \mathrm{von} 1,5 \mathrm{~g}$ Phlorhizin, von da an regelmässig alle 8 Stunden.

Nahrung: alle 8 Stunden $250 \mathrm{~g}$ Fleisch, $10 \mathrm{~g}$ Fett, $\mathrm{H}_{2} \mathrm{O}$. (S. Tabelle.)

\begin{tabular}{|c|c|c|c|c|c|c|c|c|c|c|c|c|}
\hline \multirow{2}{*}{$\begin{array}{c}\text { Datum } \\
1901\end{array}$} & \multirow{2}{*}{. } & \multirow{2}{*}{$\mathrm{N}$} & \multicolumn{3}{|c|}{ Zucker } & \multicolumn{2}{|c|}{ Linksdrehg. } & \multirow{2}{*}{$\ddot{\theta}$} & \multirow{2}{*}{ 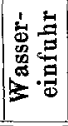 } & \multirow{2}{*}{ 最总 } & \multirow{2}{*}{ 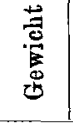 } & \multirow{2}{*}{$\begin{array}{l}\text { Bemer- } \\
\text { kungen }\end{array}$} \\
\hline & & & Pol. & $\begin{array}{l}\text { Sa. } \\
2, \text { Per. }\end{array}$ & $\begin{array}{l}\text { Red. } \mathbf{n} \\
\text { Allinn }\end{array}$ & $\begin{array}{l}\text { n. Ver- } \\
\text { gahrg. }\end{array}$ & $\begin{array}{l}\text { ber.a.g } \\
\text { Glucos. }\end{array}$ & & & & & \\
\hline \multirow{4}{*}{$\begin{array}{l}\text { 20. V. } \\
\text { 21. V. }\end{array}$} & 3 & 6,5 & 3 & \multirow{2}{*}{66,30} & & \multicolumn{2}{|l|}{$-0,2$} & 4.9 & 150 & \multirow{2}{*}{$\begin{array}{l}330 \\
330\end{array}$} & \multirow{2}{*}{$\begin{array}{l}24,0 \\
23,6\end{array}$} & \multirow{10}{*}{$\begin{array}{c}10 \mathrm{~g} \\
\text { Campher }\end{array}$} \\
\hline & 1 & & 34, & & & $-0,1$ & & 3,9 & 235 & & & \\
\hline & 2 & 8, & 34, & \multirow{2}{*}{65,64} & & -0 , & & 3,9 & 255 & 330 & & \\
\hline & 3 & & & & & & & & 50 & 340 & & \\
\hline \multirow[t]{3}{*}{ 22. V. } & 1 & 9,07 & 34,32 & \multirow{2}{*}{64,74} & 37 & $-0,2$ & & 3,8 & 250 & 345 & \multirow{2}{*}{23,2} & \\
\hline & 2 & 8 , & 30 & & 31 & -0 & & 3,7 & 275 & 340 & & \\
\hline & 3 & 11,06 & 46,80 & 84,24 & 43,9 & $-0,8$ & 4,8 & 4,2 & 900 & 620 & & \\
\hline & 1 & 9,9 & 37,44 & & 32,4 & $-1,0$ & 6,4 & 3,9 & - & 420 & 23,15 & \\
\hline & $\begin{array}{l}2 \\
3\end{array}$ & 9,7 & 25,74 & 55,38 & 30,9 & $-0,1$ & & 2,7 & $\mathbf{4 5}$ & 360 & & \\
\hline & 3 & 9, & 29,0 & & 34,0 & -0 & & 3,3 & 200 & 1330 & & \\
\hline
\end{tabular}

Der Verlauf des Versuches bestätigt schlagend das Ergebniss der bisherigen. Er ist insofern als der eleganteste za bezeichnen,

1) Es gelingt übrigens auch nicht, wie mich zu diesem Zweck angestellte Versuche belehrten, durch wochenlang fortgesetzte Injection der eben noch Glycosurie hervorrufenden Gabe das Thier an Phlorhizin zu gewöhnen. 
als jede grobe Schwankung des Stickstoffwechsels wegfällt. In der ersten Periode nach Campherzufuhr steigt der N-Umsatz, mit ihm die Zuckerausscheidung und zwar trotz des Auftretens von Camphoglucuronsäure entsprechend $4,8 \mathrm{~g}$ Zucker mehr als der $\mathrm{N}$, sodass $\mathrm{D}: \mathrm{N}$ den höchsten Werth der ganzen Reihe erreicht. In der nächsten Periode (23. V. 1) ist $\mathrm{D}: \mathrm{N}$ eben so hoch, wie vor der Campherzufuhr, erst dann, als tiberhaupt keine Glucuronsäure mehr ausgeschieden wurde, sank das Verhältniss in der jedesmal beobachteten Weise ab.

Es lässt sich also unter der oben gemachten Einschränkungaus diesen Versuchen schliessen, dassweder die M uttersubstanzen fur die Glucuronsäure dieselben sind wie für den Zucker, noch dass die Säure aus diesem selbst entsteht.

Ferner hat sich ergeben, dass Campher zu einem von der Glucuronsäurebildung unabhängigen Sinken der Zuckerausscheidung fïhrt.

Im folgenden Versuch prtifte ich, ob es gelänge, durch dauernde, intensive Camphervergiftung den Diabetes vielleicht ganz zu unterdrücken.

\section{Versuch 5.}

Männlicher Hund, $7,1 \mathrm{~kg}$ schwer, wird am ersten Versuchstag mit $3 \mathrm{~g}$, von da an mit von Tag zu Tag um $2 \mathrm{~g}$ steigenden Dosen von Campher gefuttert.

29. X. 1900 erbält er $38 \mathrm{~g}$ Campher (20. Versuchstag).

$30 . X \cdot 1900=\quad 40=\quad=(21 .=)$.

31. X. 1900 Harnmenge $670 \mathrm{ccm}$ auf $1000 \mathrm{ccm}$ aufgef.

Drehung vor Verg.: 4,8 Proc. Linksdr. nach Verg.: 4,6 Proc. Linksdr.

$40 \mathrm{~g}$ Campher and Abends $0,3 \mathrm{~g}$ Phlorhizin subcut.

1. XI. 1900 Harnmenge 470 anf $500 \mathrm{ccm}$ aufgef.

Drehung vor Verg.: 2,3 Proc. Linksdr. nach Verg.: 4,4 Proc. Linksdr.

daraus berechnet sich eine Glucoseausscheidung von $10,5 \mathrm{~g}$.

Es wurde also trotz starker und lange dauernder Camphervergiftung nach Einfuhr einer geringen Phlorhizinmenge eine reichliche Zuckerausscheidung erzielt.

Suchen wir uns über die Wirkungsart des Camphers bei Phlorhizindiabetes eine Vorstellung zu machen, so müssen wir in erster Linie an eine Beeinflussung der Niere denken, die nach der wohl bestgegründeten Anschauung der Angriffspunkt des Phlorhizins ist. Man hat ja auch bereits gefunden, dass bei experimentell erzeugter Nephritis (Chromsäure, Cantharidin) die Phlorhizinzuckerausscheidung 
fällt. Bei diesen Vergiftungen handelt es sich um grob mechanische Läsion der Niere, die dabei anch für andere Stoffe weniger durchlässig wird. Dem Campher kommt im Gegensatz zu den genannten Giften eine anatomisch nachweisbare Wirkung auf die Niere nicht zu. Die Nieren zeigen weder makroskopisch noch mikroskopisch die geringste Veränderung; während des Lebens trat nie Albuminurie auf. Damit ist eine Nierenwirkung des Camphers natürlich nicht ausgeschlossen, sie braucht nur für unsre Methodik nicht nachweisbar zu sein. Jedenfalls beansprucht die Verfolgung der Campherwirkung ein besonderes Interesse, doch scheint es mir an Angriffspunkten für ihre experimentelle Verfolgung vorläufig zu feblen.

\section{A n h ang.}

a) Nach Blumenthal1) soll das Eiweiss, woraus beim Phlorhizindiabetes Zucker gebildet wird, besonders phosphorreich sein. Rumpf konnte diese Angabe nicht bestätigen, die nach den Untersuchungen in Voit's Laboratorium, wobei sich der Umsatz besonders phosphorreicher Gewebe an den Ausscheidungen nicht erkennen liess, von vornherein recht fragwürdig erschien. Auch meine Untersuchungen liefern keine Stütze für ihre Richtigkeit.

Bei den fleischgefütterten Hunden beträgt der Factor $\mathrm{N}: \mathrm{P}_{2} \mathrm{O}_{5}$ im Harn 8,1.

In Versuch 1 beträgt der Factor im Mittel 7,7

$=2 "=2=9,0$

$=\quad 3$ schwankt der Factor zwischen 6,7 und 7,0 .

b) Tabelle der in den einzelnen Versuchen für die Ausscheidung: der Camphoglucuronsäure gefundene Werthe.

\begin{tabular}{|c|c|c|c|c|c|c|c|}
\hline \multirow[b]{2}{*}{ Versuch } & \multirow{2}{*}{$\begin{array}{l}\text { Campher- } \\
\text { einfuhr } \\
\text { in } g\end{array}$} & \multicolumn{2}{|c|}{$\begin{array}{c}\text { Linksdrehung - Procent } \\
\text { berechnet }\end{array}$} & \multirow{2}{*}{$\begin{array}{l}\text { Campho- } \\
\text { glucuron- } \\
\text { säure } \\
\text { absolut }\end{array}$} & \multirow{2}{*}{$\begin{array}{l}\text { Campho- } \\
\text { glucuron- } \\
\text { säure } \\
\text { erwartet }\end{array}$} & \multirow{2}{*}{\multicolumn{2}{|c|}{ 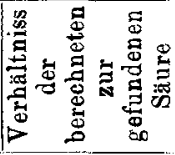 }} \\
\hline & & $\begin{array}{l}\text { als } \\
\text { Glucose }\end{array}$ & $\begin{array}{c}\text { als } \\
\text { Campho- } \\
\text { glucuronsäure }\end{array}$ & & & & \\
\hline 1 & 35 & 0,8 & 1,28 & 76,8 & 84 & 92 & Proc. \\
\hline $2 a$ & 20 & 0,9 & 1,44 & 57,6 & 48 & 120 & $=$ \\
\hline$b$ & 25 & 1,1 & 1,76 & 70,4 & 60 & 117 & $=$ \\
\hline $3 a$ & 15 & 0,6 & 0,96 & 38,4 & 36 & 107 & $=$ \\
\hline b & 20 & 0,86 & 1,38 & 55,2 & 48 & 115 & $=$ \\
\hline 5 & 10 & 0,7 & 1,12 & 22,5 & 24 & 96 & $=$ \\
\hline
\end{tabular}

1) Dtsch. med. Wochenschr. 1899. S. 814. 